\title{
On the Rank Distance of Cyclic codes
}

\author{
U.Sripati and B.Sundar Rajan ${ }^{1}$ \\ ECE Dept.,Indian Institute of Science \\ Bangalore,-560012,India \\ \{sripatieprotocol. , bsrajane\}ece.iisc.ernet.in
}

\begin{abstract}
We study the rank-distance of primitive length $\left(n=q^{m}-1\right)$ linear cyclic codes over $F_{q^{m}}$ using the Discrete Fourier Transform (DFT) description of these codes.
\end{abstract}

\section{INTRODUCTION}

Let $\mathcal{C}$ be an $[n, k]$ linear code over $F_{q^{m}}$. For any pair of codewords $\overrightarrow{c_{1}}, \overrightarrow{c_{2}} \in \mathcal{C}$, the rank distance between them is defined to be the rank over $F_{q}$ of the $m \times n$ matrix corresponding to $\overrightarrow{c_{1}}-\overrightarrow{c_{2}}$ obtained by expanding each entry of $\overrightarrow{c_{1}}-\overrightarrow{c_{2}}$ as an $m$-tuple along a basis of $F_{q^{m}}$ over $F_{q}$ and is denoted by $\operatorname{Rank}_{q}\left(\overrightarrow{c_{1}}-\overrightarrow{c_{2}}\right)$ [2]. The rank of $\mathcal{C}$, denoted by $\operatorname{Rank}_{q}(\mathcal{C})$ is defined as the minimum of $\operatorname{Rank}_{q}\left(\overrightarrow{c_{1}}-\overrightarrow{c_{2}}\right)$ over all possible pairs of distinct codewords. Rank-distance codes over finite fields have been studied by several authors for applications in storage devices and more recently for applications in SpaceTime coding. These studies are for the general class of linear codes and hence specific results like expressions or bounds for the rank of the code given by the description of the code are not known. In view of this, in this paper, we focus on primitive length $\left(n=q^{m}-1\right)$ cyclic codes over $q^{m}$ and obtain expressions and upper bounds for the rank-distance of few sub classes of these codes. Note that this class of codes includes the well known Reed-Solomon codes.

DFT Description of cyclic codes:[1] Let $\vec{a}=$ $\left(a_{0}, a_{1}, \cdots, a_{n-1}\right) \in F_{q^{m}}^{n}$ where $q$ is power of a prime and $(n, q)=1$ (i.e., $n$ and $q$ are relatively prime-which is assumed to hold throughout the paper). Also let $r$ be the smallest positive integer such that $n \mid q^{m r}-1$ and let $\alpha \in F_{q^{m r}}$ an element of order $n$. The DFT of $\vec{a}$ is defined to be the vector $\vec{A}=\left(A_{0}, A_{1}, \cdots, A_{n-1}\right) \in F_{q^{m r}}^{n}$ given by $A_{j}=$ $\sum_{i=0}^{n-1} \alpha^{i j} a_{i}, \quad j=0,1, \cdots, n-1$. Denoting the set $\{0,1, \cdots, n-1\}$ by $I_{n}$, for any $j \in I_{n}$, for any divisor $d$ of $m$ the $q^{d}$-cyclotomic coset of $j$ modulo $n$ is defined to be the set $[j]^{d}=\left\{i \in I_{n} \mid j=i q^{d t}(\bmod n)\right.$ for some $\left.t \geq 0\right\}$ and we will denote the cardinality of this set by $e_{j}^{(d)}$. When $d=1$ we denote the $q$-cyclotomic coset of $j$ modulo $n$ by $[j]$ and its cardinality by $e_{j}$. Simply by "cyclotomic coset", we mean a " $q$-cyclotomic coset". For a code $\mathcal{C}$ we say $A_{j}$ takes values from $\left\{\sum_{i=0}^{n-1} \alpha^{i j} a_{i} \mid \vec{a} \in \mathcal{C}\right\} \subset F_{q^{m r}}$. For linear cyclic codes $A_{j}$ takes values from $\{0\}$ or $F_{q^{m e_{j}^{(m)}}}$ and transform components in different $q^{m}$-cyclotomic cosets are unrelated and within a $q^{m}$ cyclotomic coset are related by $A_{j q^{m}}=A_{j}^{q^{m}}$ (called conjugacy constraints). The transform components taking values from $F_{m e_{j}^{(m)}}$ are called free spectral components. Note that for length $n=q^{m}-1$ codes over $F_{q}$ each cyclotomic coset is a singleton set and there is no conjugacy constraint. Also we will be using the following sets frequently throughout that are

${ }^{1}$ This work was partly supported by the DRDO-IISc program on Mathematical Engineering through a grant to B.S.Rajan closely related to the cyclotomic cosets: $\alpha_{[j]^{d}}=\left\{\alpha^{i} \mid i \in[j]^{d}\right\}$ and $A_{[j]^{d}}=\left\{A_{i} \mid i \in[j]^{d}\right\}$.

II. RANK-DISTANCE OF $q^{m}-1$ LENGTH CYCLIC CODES The following proposition is straightforward.

Proposition 1: Let $\mathcal{C}$ be a cyclic code of length $n$ over $F_{q^{m}}$ with $A_{0}$ being one of the free transform domain components. Then $\operatorname{Rank}_{q}(\mathcal{C})=1$.

Identifying codewords with all the components from a proper subfield of $F_{q^{m}}$ leads to

Proposition 2: Let a cyclic code $\mathcal{C}$ of length $n=q^{m}-1$ over $F_{q^{m}}$ be characterized by all the free transform domain components in the union of sets of the form $A_{[j]^{d}}$ for some values of $j$ and all other transform domain components 0 . Then $\operatorname{Rank}_{q}(\mathcal{C}) \leq d$.

Theorem 1: Let $\mathcal{C}$ be a cyclic code of length $n=q^{m}-1$ over $F_{q^{m}}$ such that the transform domain component $A_{j q^{s}} \in$ $A_{[j]},\left(0 \leq s \leq e_{j}-1\right)$ is the only free component and all other transform domain components are constrained to zero. Then $\operatorname{Rank}_{q}(\mathcal{C})=e_{j}$

Theorem 2: Consider a cyclic code $\mathcal{C}$ of length $n=$ $q^{m}-1$ over $F_{q^{m}}$ for which the two transform domain components $A_{j q^{r}}$ and $A_{j q^{r+s}}$ are free, (s denotes the separation between the two free transform domain components) $\left(0 \leq r \leq e_{j}-2\right),\left(1 \leq s \leq e_{j}-1\right)$ and all other transform domain components be constrained to 0 . Then $\operatorname{Rank}_{q}(\mathcal{C})=$ $\left(e_{j}-\operatorname{gcd}\left(s, e_{j}\right)\right)$. Moreover, the rank weight distribution of $\mathcal{C}$ is given by $N_{0}=1 ; N_{e_{j}-g c d\left(s, e_{j}\right)}=\tau\left(q^{m}-1\right) ; N_{e_{j}}=$ $q^{2 m}-N_{e_{j}-g c d\left(s, e_{j}\right)}-1$, where $N_{i}$ denotes the number of codewords with rank weight $i$ and $\tau$ is the number of elements of $F_{q^{m}}$ that have their $\left(q^{s}-1\right)$-th root in $F_{q^{e_{j}}}$.

An Upper Bound: We can make use of Theorem 2 to obtain an upper bound on the Rank of a code $\mathcal{C}$ characterized by more than two free transform domain components drawn from the same cyclotomic coset as follows:

Let $A_{j q^{s_{1}}}, A_{j q^{s_{2}}}, \cdots, A_{j q^{s_{1}}}, \cdots, A_{j q^{s_{i}}}, \cdots, A_{j q^{s_{k}}}$ be the $k$ free transform domain components. Then

$$
\begin{gathered}
\operatorname{Rank}_{q}(\mathcal{C}) \leq \min _{(i, l),(i>l)}\left\{e_{j}-\operatorname{gcd}\left[\left(s_{i}-s_{l}\right), e_{j}\right]\right\}= \\
e_{j}-\max _{(i, l),(i>l)}\left\{\operatorname{gcd}\left[\left(s_{i}-s_{j}\right), e_{j}\right]\right\} .
\end{gathered}
$$

The following theorem identifies certain situations leading to a tighter upper bound.

Theorem 3: Let $A_{j q^{s_{1}}}, A_{j q^{s_{2}}}, \cdots, A_{j q^{s_{k}}}$ be the set of all free transform domain components of $\mathcal{C}$ where $s_{1}, s_{2}, \cdots, s_{k}$ with $s_{1}<s_{2}<\cdots<s_{k-1}<s_{k} \leq e_{j}-1$ are related by $s_{2}-s_{1}=$ $s_{3}-s_{2}=\cdots=s_{k}-s_{k-1}=s_{1}-s_{k}=s^{\prime}$ modulo $e_{j}$, where $s^{\prime}$ divides $e_{j}$. Then $\operatorname{Rank}_{q}(\mathcal{C}) \leq s^{\prime}$.

\section{REFERENCES}

[1] R.E.Blahut, Theory and Practice of Error Control Codes, Addison Wesley, 1983.

[2] E. M. Gabidulin, "Theory of Codes with Maximum Rank Distance," Problemy Peredachi Informatsii, 21, 99.3-14, Jan.Mar.1985. 